\title{
THE SECOMOR
}

\section{A Kinematic Device Which Imitates the PERFORMANCE OF A SERIES-WOUND POLYPHASE COMMUTATOR MOTOR}

\author{
BY V. KARAPETOFF
}

Abstract of Paper

The device consists of four bars of adjustable useful length and with adjustable angles. These bars can be set in a combination to represent the vector diagram of voltages in a motor with any desired constants. By moving the bars to vary the load, complete performance characteristics of the motor can be obtained, including the speed, the torque, the power factor, etc. An additional device called the impedometer permits to take into account the impedance drop in the machine. An adjustable saturation curve made of soft wire is used in connection with the secomor, to enable one to investigate the effect of saturation. A brief graphical theory of the motor precedes the description of the secomor to make its action understandable.

\section{List of Symbols Used}

$E$ total useful voltage (or counter e. m. f.) of the machine.

$E_{1} \quad$ stator e. m. f.

$E_{2}$ rotor e. m. f. at standstill.

$I \quad$ current in the machine without the transformer.

$I_{1}$ stator current with the transformer (Fig. 11).

$I_{2} \quad$ rotor current with the transformer (Fig. 11)

$I_{m} \quad$ exciting current of the machine (Fig. 6)

$I_{0}$ exciting current of the series transformer (Fig. 11).

$M$ exciting $\mathrm{m}$. m. f. of the machine (Fig. 6).

$M_{1}$ stator m. m. f.

$M_{2}$ rotor m. m. f.

$n_{1} \quad$ effective stator turns

$n_{2}$ effective rotor turns.

$P$ line voltage.

$r \quad$ resistance of the motor circuit

$s$ slip expressed as a fraction.

$u$ ratio $n_{2} / n_{1}$ of the effective rotor turns to the effective stator turns. 
$x \quad$ leakage reactance of the machine.

$Z_{1} \quad$ primary leakage impedance of the series transformer.

$Z_{2}$ secondary leakage impedance of the series transformer.

$z \quad$ leakage impedance of the machine.

$\alpha$ brush shift angle measured from the opposition setting.

$\alpha^{\prime}$ same, modified by the transformer magnetizing current, Fig. 12.

$\beta \quad$ angles defined in Fig. 6

$\left.\gamma_{\delta}\right\}$ an angle defined in Fig. 12.

$\Phi$ useful flux in the air-gap.

$\phi$ phase angle of the machine.

\section{A. Introduction}

THE MEANING of the Name Secomor: An abbreviation of the words "series commutator motor".

What the Secomor is. A combination of movable and adjustable bars (Fig. 9) which can be set to represent a vector diagram of voltages, currents, m.m.f's and fluxes in a serieswound polyphase commutator motor with any desired constants.

The Purposes of the Device. (1) To enable a designer to select the best electrical constants and to "test" a motor before it has been actually built; (2) To take the place of a complicated circle diagram which does not hold true anyway when the iron is saturated; (3) To do away with an involved analytical theory because it is often difficult to see the effect of separate factors upon the performance characteristics, and because it takes considerable mathematical skill to deduce the equations of the various loci; (4) To add the judgment of the eye and the skill of the hands to the purely mental ability in selecting the constants of a motor for a desired performance, or in judging the characteristics for assumed constants; (5) To enable an investigator or a student to familiarize himself with the motor as if he had one available for tests.

The performance curves that the secomor enables one to draw: Current, torque, speed, input, output, efficiency, power factor, magnetizing current. These may be obtained just as easily at a constant applied voltage as at a constant current, or under any variable conditions of service.

The factors which may be taken into account and varied at will in the secomor: The ratio of the primary to the secondary ampere turns; the ratio of either one to the exciting m.m.f.; the angle of brush shift; the ohmic drop and the reactive drop; 
saturation in iron; variable core-loss and friction; reaction of short-circuited armature coils upon the exciting current.

\section{B. General Properties of the Motor}

A detailed description of the motor, its field of application, its performance characteristics and a complete theory, both graphical and analytical, will be found in E. Arnold's "Die Wechselstromtechnik", (1912) Vol. V, Part 2, Chap. 2. See also the bibliography below.

Diagrams of connections are shown in Figs. 1, 2 and 3. The stator is phase-wound and is similar to that of an induction motor. The rotor is like a d-c. armature with a commutator. With a multiple armature winding the number of brushes per

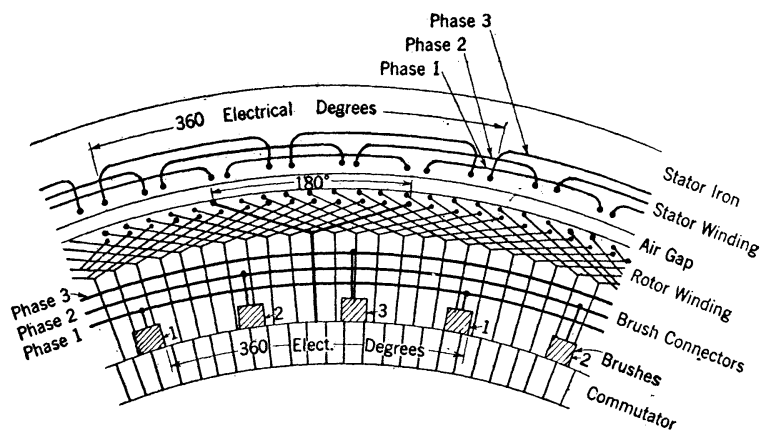

Fig. 1-The Stator, the Armature Winding and the Brushes in a Three-Phase Commutator Motor

pair of poles is equal to the number of phases; with a two-circuit winding it is considerably less.

The rotor is connected in series with the stator, either directly (Fig. 2), or through a current transformer (Fig. 3). In the latter case the stator may be either star or mesh connected.

Characteristics and the field of application. The motor has a speed-torque characteristic similar to that of a d-c. or singlephase series motor. As the load increases the speed decreases. At no-load there is a tendency to run away. Roughly speaking, one may consider the motor as a combination of three singlephase series-connected motors built on the same frame. On account of the mutual action between the phases such a view is not quantitatively correct. The motor may be used for crane service and in other applications in which it may replace a series-wound d-c. motor. A peculiar field of its own is in cascade 
connection with an induction motor, as a counter e.m.f. arrangement for speed control. This is useful in mine-hoist work and in rolling mills; see Arnold, p. 266. A shunt-connected polyphase commutator motor is also suitable for cascade connection.*

Advantages of a series transformer (Fig. 3): (a) The stator

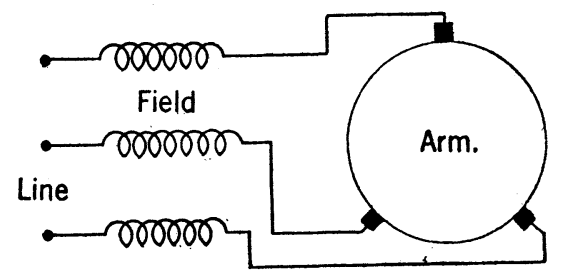

Fig. 2-Three-Phase Series Commutator Motor-the Armature Supplied at the Line Voltage

may be wound for a comparatively high voltage, e.g., 2200 volts; (b) The rotor may be wound for such a value of current as to give the best commutation; (c) When the machine is used as a generator, for example in regenerative braking, there is a possibility of a harmful direct current or of low-frequency alternating currents produced in the armature, due to self-excitation.

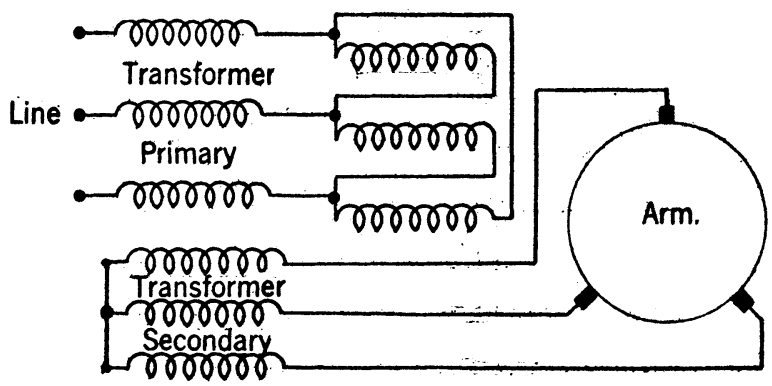

Fig. 3-Three-Phase Series Commutator Motor-the Armature Supplied Through a Transformer

By saturating the current transformer this tendency is counteracted; see Arnold, p. 64 . (d) By properly selecting the saturation and the magnetizing current of the transformer the speed above synchronism may be controlled to some extent, so that the motor does not run away when the load is removed.

*See J. D. Wright, General Electric Review, 1916, p. 104. 
Four fundamental properties of an armature connected to a polyphase a-c. circuit through a commutator and brushes, Figs. 1 and 2.

(a) The currents in the armature conductors are of the same frequency as the line currents, independent of the speed of rotation. Call the armature coils (Fig. 1) between two adjacent brushes "a group". The function of the commutator is to transfer the coils in succession from one group to the next. But a new coil is always substituted in place of the one transferred, so that the group persists and forms a steady path for the line currents. This, of course, does not apply to the coils undergoing commutation, in which high-frequency currents are induced.

(b) The voltages induced in the armature conductors are always of the same frequency as that of the stator flux which induces them. The relative speed of rotation of the flux and armature influences only the magnitude of the induced voltage but not its frequency. Consider a d-c. machine in which the field current and the flux are periodically varied. The e.m.f. induced between the brushes undergoes corresponding fluctuations. If the field be varied harmonically, the induced e.m.f. will also vary at the same frequency according to a sine law. Speeding up the armature or slowing it down will influence the value of the induced voltage, but its frequency is always that of the flux. With a constant flux the voltage is also constant. Similarly, in an a-c. motor the instantaneous voltage induced in a group of coils between adjacent brushes is proportional to the instantaneous value of the stator flux and to the speed of rotation. The stator flux varies at the impressed frequency, and so does the induced voltage. This simple property of armature currents and voltages makes it possible to use vector diagrams at any speed of rotation. It will be remembered that in an induction motor (without commutator) the frequency of the secondary currents depends upon the speed of the rotor.

(c) The magnetomotive force and the flux due to the armature currents are nearly the same whether the armature is revolving or is at rest, provided that the armature current is kept constant. We have seen above that the groups of armature coils between the brushes are always stationary, and the currents that flow through these groups are of the same frequency as the line currents. Hence, the m.m.fs. of the individual groups are sta- 
tionary in space at all speeds of the armature. The combined action of several groups of coils produces a revolving field, same as in the stator winding, and this field glides synchronously in the air-gap with respect to the brushes, no matter what the speed of the armature might be. The m.m.f. due to highfrequency currents in the coils undergoing commutation is a disturbing factor, being a function of speed, current, stator flux, etc., but its effect is usually small. For its computation see Arnold, pp. 23 and 56.

(d) The leakage reactance of a commutated armature is a function of its speed, unlike that of ordinary a-c. windings. Two kinds of leakage fluxes may be distinguished, (1) those linking with individual armature conductors or with groups of conductors belonging to the same phase, and (2) those linking with conductors belonging to different phases. The first named fluxes (phase leakage) are carried around with the conductors and cause a reactance drop which is independent of the speed of rotation of the armature. The latter fluxes (interlinked leakage) in combination form a true revolving flux which glides synchronously in the air gap whether the armature is standing still or revolving at any speed.

The effect of the interlinked leakage flux depends upon the slip of the armature. At synchronous speed the armature conductors do not cut this flux at all, and the only reactance is that due to the phase fluxes which travel with the conductors. At standstill the interlinked leakage flux exerts its full effect. At speeds above synchronism the armature conductors cut this flux in the opposite direction, so that it partly compensates for the effect of local or phase fluxes. At a certain synchronous speed the total armature reactance becomes zero, and beyond that speed it becomes negative. Whatever the theory of the phenomenon, actual experiments show that the reactance of a commutated armature winding decreases with increasing speed. See Arnold, p. 20.

Brush shift and the m.m.fs. The alternating currents in the three stator phases together produce an m.m.f. $M_{1}$ (Fig. 4) of constant amplitude. This m.m.f. is distributed in space approximately according to a sine law, and it glides synchronously along the air gap. The action is the same as in the stator of an ordinary polyphase induction motor; see for example the theory as treated in the author's "Magnetic Circuit", p. 128. The three rotor currents together produce a similar gliding magneto- 
motive force $M_{2}$, whether the armature is revolving or stationary. Its position in space with respect to $M_{1}$ depends only upon the angle of brush shift and is independent of the value of the

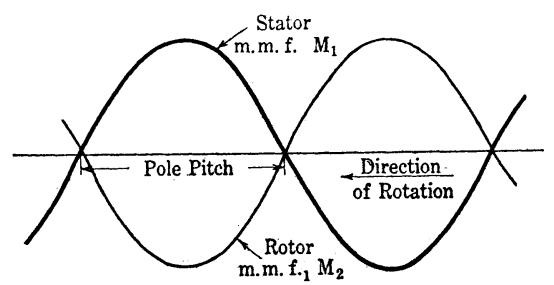

Fig. 4-Stator and Rotor m. M. F's in Space-the Brushes Are in the Neutral Position

current or of the speed of rotation. This is because the m.m.fs. in a commutated armature are the same as if the armature were at rest (see proof above). In a certain position of the brushes

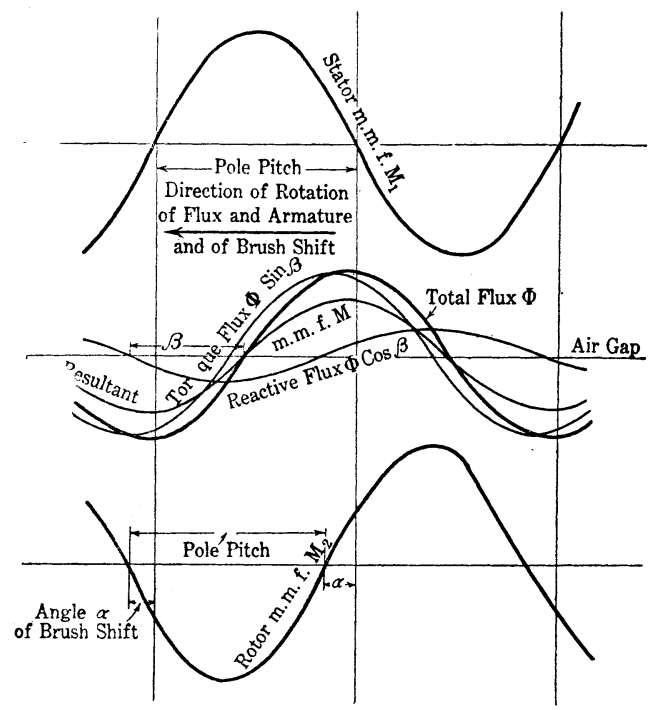

Fig. 5-Stator and Rotor M. M. F's. AND the Resultant Fi.ux in Space-the Brushes are Shifted by an Angle $\alpha$ in the Direction of Rotation

called neutral the stator and the rotor m.m.fs. are in phase opposition in space (Fig. 4). In this paper the angle of brush shift $\alpha$ is measured from this position. Arnold measures the brush shift angle $\rho$ from the position at which $M_{1}$ and $M_{2}$ are 
in phase coincidence. Hence, his $\rho=180$ deg. $-\alpha$. In the secomor it is more convenient to deal with an acute angle $\alpha$ than with an obtuse angle $\rho$.

The Torque. In the neutral position of the brushes (Fig. 4) the mechanical force between the stator and the rotor is simply a radial repulsion, and the motor develops no torque. Let now the brushes be shifted by an angle $\alpha$ (Fig. 5) in the direction of rotation of both m.m.fs. The resultant m.m.f. $M$ in the airgap is equal to the sum of $M_{1}$ and $M_{2}$, and the flux $\Phi$ which $M$ produces is out of phase with $M_{1}$ and $M_{2}$. The relations shown in Fig. 5 are also indicated vectorially in Fig. 6 , which is a space (not time) diagram. The actual air-gap flux $\Phi$ may be resolved into two space components, one in phase (or phase opposition) with $M_{2}$, the other in quadrature with it. These components

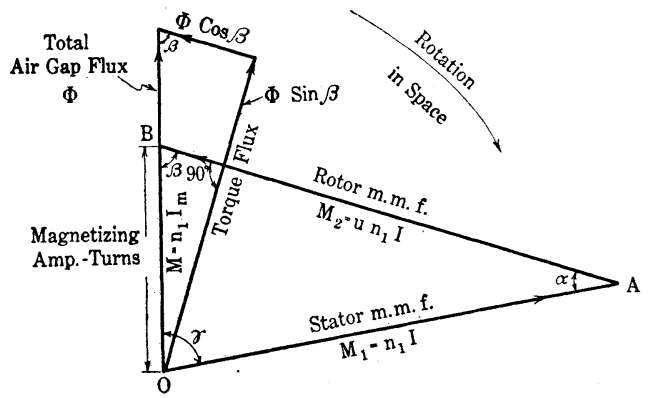

Fig. 6-A Vector Representation of the m. m F's. and Flux Shown in Fig. 5-Space Diagram

are marked $\Phi \cos \beta$ and $\Phi \sin \beta$ respectively. The in-phase component $\Phi \cos \beta$ increases or reduces the fictitious flux due to the rotor winding itself, and produces no torque with the armature currents. The useful torque is due entirely to the interaction between the quadrature component $\Phi \sin \beta$ of the air-gap flux and the armature m.m.f. $M_{2}$. There is a similar relationship in a d-c. machine with the brushes in the neutral, no torque being produced between the armature conductors and the flux of armature reaction. The useful flux must be in space quadrature with that which the armature windings themselves excite.

Direction of Rotation of the Armature. By marking the directions of the currents and fluxes in Fig. 5 and applying the familiar right-hand screw rule one finds that the torque is exerted upon the armature conductors in the direction in which the 
brushes are shifted from the neutral. A simple way to check this fact is to consider the fictitious fluxes due to $M_{1}$ and $M_{2}$ acting alone. Since the m.m.fs. are nearly in phase opposition, these fluxes repel each other, and the armature tends to carry its m.m.f. away from $M_{1}$, by moving to the left. The stationary brushes fix definite groups of armature conductors in space, so that the armature moves away steadily without coming into a position of stable equilibrium. If the brushes were shifted in the opposite direction, the direction of rotation would be reversed. It is of importance to note that the characteristics of the motor are entirely different for the two directions of

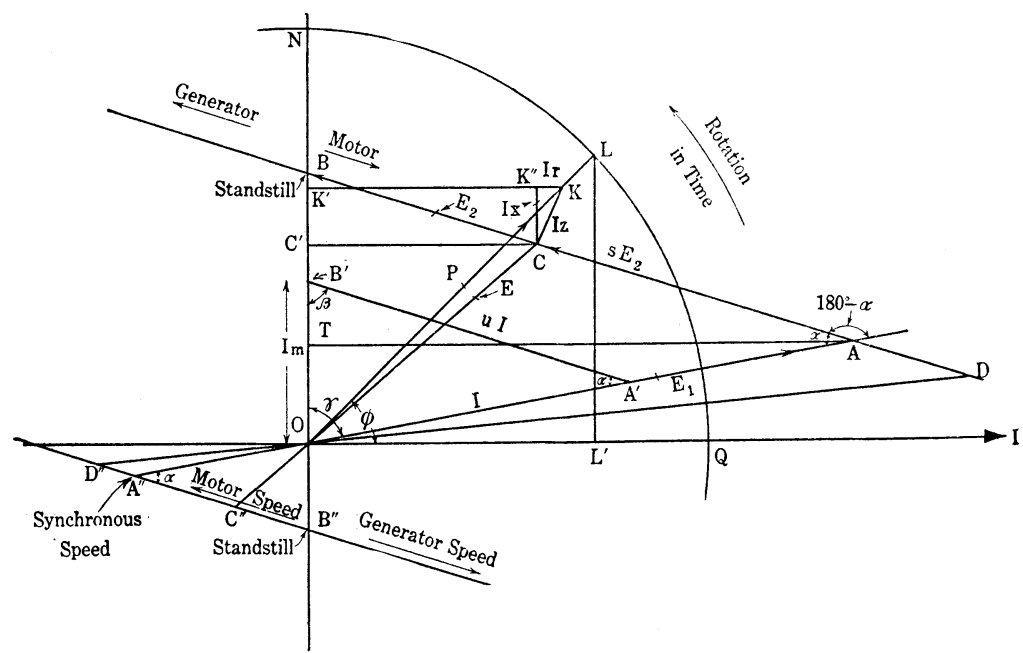

Fig. 7-Time Diagram of Voltages and Data for Performance Characteristics

rotation. In the first case the armature conductors revolve in the direction of the flux, so that the rotor e.m.f. at synchronism becomes zero, and then its sign is reversed. In the second case the rotor e.m.f. increases indefinitely with the speed of rotation. In practise the first combination has been used so far, and is the only one considered in this paper.

\section{Time Diagram of E.M.Fs.}

Consider the motor running at a given current, which is kept constant and let the speed vary, the terminal voltage being adjusted for each speed. The e.m.fs. $M_{1}$ and $M_{2}$ are constant and 
so is the resultant flux $\Phi$, so that Fig. 6 applies at all speeds. The flux $\Phi$ induces certain e.m.fs. in the stator and rotor windings, and these e.m.fs. are shown in Fig. 7.

Fig. 7 is a time diagram, and it shows the applied voltage $O K=P$ consumed, in three parts: (1) $O A=E_{1}$ is that part of the applied terminal voltage which is equal and opposite to the e.m.f. induced by flux $\Phi$ in the stator windings. For the sake of brevity $E_{1}$ is further referred to as the stator voltage. (2) $A C=s E_{2}$ is the voltage consumed in the rotor, in opposition to the e.m.f. induced by flux $\Phi$. (3) $C K=I z$ is the impedance drop in the windings of the machine and in the brushes.

The voltage induced in the rotor is proportional to per cent slip, being zero at synchronism. Let $A B=E_{2}$ be the voltage which balances that induced in the rotor at standstill. With the brushes in the neutral, $E_{2}$ leads $E_{1}$ in time by $180 \mathrm{deg}$. Shifting the brushes forward by an angle $\alpha$ is equivalent to retarding the armature with respect to the revolving flux by the same angle, so that $E_{2}$ leads $E_{1}$ by $(180 \mathrm{deg} .-\alpha)$. At a slip $s$, the secondary voltage is no more $A B$, but is $A C=s E_{2}$. The resultant voltage $E=O C$ balances the total counter e.m.f. of the machine.

In an ideal motor, without internal resistance or reactance, $O C$ is identical with the terminal voltage $P$. As the speed increases from zero to synchronism, with the current kept constant, point $C$ moves along $B A$ from $B$ to $A$. Above synchronism the sign of $E_{2}$ is reversed and point $C$ moves further towards $D$. For points to the left of $B$ the machine acts as a generator.

In an ideal motor no power is consumed at standstill so that the applied voltage $O B$ at the speed zero must be in leading quadrature with the horizontal current vector $I$, which is the reference vector. Thus $O B$ in the diagram must be vertical. As the speed increases the phase angle between $O C$ and $I$ decreases. By continuing $B D$ further to the right to its intersection with $I$, a super-synchronous speed is found at which the applied voltage is in phase with the current. Beyond that speed, the motor takes in a leading current.

The Triangles of Voltages and of m.m.fs. are Similar. In the construction of the secomor use is made of the fact that the triangles $O A B$ in Figs. 6 and 7 are similar. The two m.m.fs., $M_{1}$ and $M_{2}$, being produced by the same current are displaced in space by 180 deg. $-\alpha$, where $\alpha$ is the brush shift angle measured from the opposition point (Fig. 5). Similarly, the two voltages, 
$E_{1}$ and $E_{2}$, being induced by the same flux $\Phi$, are displaced in time by 180 deg. $-\alpha$. The ratio of $M_{1}$ to $M_{2}$ is equal to that of the effective numbers of turns in the stator and in the rotor; the ratio of $E_{1}$ to $E_{2}$ is equal to the same ratio of turns. Thus, the two triangles have an equal angle between two proportional sides, and therefore are similar. This important relationship permits to incorporate the m.m.f. triangle $O A^{\prime} B^{\prime}$ in the time diagram (Fig. 7) and to do away with a separate space diagram. To take into account the m.m.f. of the short-circuited coils undergoing commutation a slight correction is necessary; see Arnold, p. 56.

Leakage Inductance. Both the stator and the rotor windings are linked with leakage fluxes, and the corresponding reactances cause a voltage drop in time quadrature with the current. The effect of the reactance is shown in Fig. 7 in the usual way, by adding a vector $I x$ to the voltage $O C$. As is explained above, the total armature reactance depends upon the speed of the machine, so that the vector $I x$ consists of two parts, one proportional to $I$ only, and the other proportional to $I$ and to the slip $s$.

Ohmic Drop, Iron Loss and Friction. The ohmic drop in the machine is taken into account in the usual way by means of the vector $I r$ in phase with $I$. In a variable-speed machine the iron loss is rather a complicated function of both the current and the speed, so that it is hardly feasible to take it into account in a vector diagram. The same is true to some extent of the friction and windage loss. The simplest way is to disregard them in the vector diagram and to correct the obtained values of input and output afterwards. One may also increase the actual ohmic resistance of the machine so as to include the iron loss in the term $I^{2} r$, and consequently in the vector $I r$, but the procedure is of doubtful accuracy and value.

The Saturation Curve. In a series-wound machine it is hardly permissible to disregard the effect of saturation in the magnetic circuit upon the performance at large values of current. The starting torque and the characteristics at low speeds are appreciably affected thereby. Let Fig. 8 represent the a-c. saturation curve of the magnetic circuit of the machine. To obtain it experimentally, the brushes are raised from the commutator, and the armature is run at the synchronous speed by some external power. The stator winding is connected to a source of variable a-c, voltage, and a series of readings is taken of the 
voltage $E_{1}$ and of the corresponding magnetizing current $I_{m}$. The values of $E_{1}$ are corrected for the ohmic drop and those of $I_{m}$ for the core loss. The corrected values give the curve shown in Fig. 8. This curve is used in connection with the diagram in Fig. 7, and also in connection with the secomor.

\section{Performance Characteristics of the Motor}

The brush shift angle $\alpha$ and the ratio $u=M_{2} / M_{1}$ of the effective rotor turns to the effective stator turns are the data to begin with. These determine the shape of the triangle $O A B$ (Fig. 7). Its position with respect to $I$ is also determined since $O B$ is perpendicular to $I$. Let $O A^{\prime} B^{\prime}$ be the m.m.f. triangle transferred from Fig. 6. Then if we make $O A^{\prime}$ equal to $I$ (in magnitude only, but not in phase), $O B^{\prime}$ will represent the magnetizing current $I_{m}$. This is because in Fig. 6 all the three

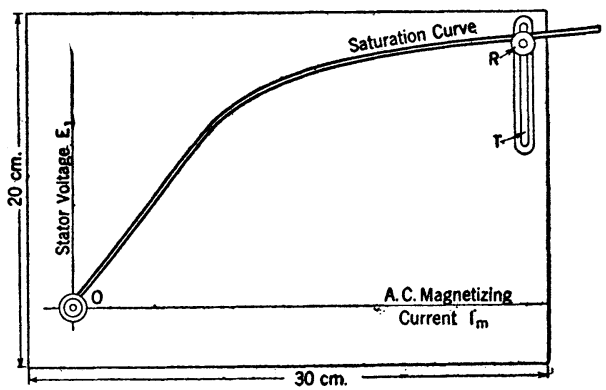

Fig. 8-An Adjustable Saturation Curve Made of Soft Wire

sides of triangle $O A B$ have a common factor $n_{1}$, that is, the number of stator turns.

From the saturation curve (Fig. 8) we get the value of $E_{1}$ corresponding to this magnetizing current and lay it off as $O A$. This determines the direction $B D$. The rest of the problem is to find such a point $C$ that the impedance triangle $I x, I r$, constructed at it, would give the desired terminal voltage $O K=P$ in magnitude. This is purely a geometrical problem which can be solved either analytically or graphically. In the secomor the point $C$ is found mechanically, by means of a few simple trials, shifting one part of the device relatively to another. Herein lies one of the principal advantages of the secomor over the analytical or the graphical method.

Speed. In Fig. 7, $B C=(1-s) E_{2}$, so that the speed of the machine $(1-s)=B C / B A$, expressed as a fraction of the 
synchronous speed. Instead of measuring every time two lengths and taking their ratio, it is convenient to draw an arbitrary straight line $A^{\prime \prime} B^{\prime \prime}$ parallel to $A B$. Produce $B O$ and $A O$ so as to get points $B^{\prime \prime}$ and $A^{\prime \prime}$, and divide $A^{\prime \prime} B^{\prime \prime}$ into 100 equal parts, beginning with zero at $B^{\prime \prime}$. Extend the divisions both ways to cover the operation above the synchronous speed, and also with the machine running backward as a generator. For any operating point, such as $C$, extend $O C$, and read per cent speed at $C^{\prime \prime}$. A proof for this construction follows directly from the similar triangles $O A B$ and $O A^{\prime \prime} B^{\prime \prime}$.

Input, Output and Efficiency. The vector $K K^{\prime}$ is the component of the applied voltage $P$ in phase with the current. Therefore $K K^{\prime} \cdot I$ represents the input into the motor per phase. To it should be added the estimated core loss in the stator. The mechanical output is proportional to the component of the counter-e.m.f., $E$, in phase with the current; hence the output is equal to $C C^{\prime} \cdot I$. The friction loss and the core loss in the armature should be subtracted from this value. The efficiency is found as the ratio of the true output to the true input.

Power Factor. The power factor, $\cos \phi$, is equal to the ratio of $K K^{\prime}$ to $O K$. A simple way to read it directly is to draw the quadrant $N L Q$ with $O$ as a center, using 100 convenient divisions, for example $100 \mathrm{~mm}$., as the radius. The intersection $L$ of $O K$ with the quadrant determines point $L^{\prime}$. Radius $O Q$ is marked with a uniform scale, zero being at $O$ to 100 at $Q$. Thus, the power factor is read at $L^{\prime}$ directly in per cent.

Torque. In a d-c. series-wound motor the torque is a function of the current and is practically independent of the speed. In the motor under consideration the torque is also independent of the speed, except for the disturbing effect of the armature coils undergoing commutation. The torque depends essentially upon the current and upon the brush shift. With the given $I$ and $\alpha$ the torque is nearly the same at standstill as at any other speed. But at standstill $A T$ represents the energy component of both the stator voltage $E_{1}$ and the rotor voltage $E_{2}$. The amount of power $A T \cdot I$ is absorbed in the stator and an identical amount is returned to the line from the rotor. Thus, $A T \cdot I$ is the torque in synchronous watts, corresponding to $I$ at any speed.

Another proof is as follows: In Arnold, on bottom of p. 41 the torque in synchronous watts is expressed as $I^{2} x_{a} u \sin \alpha$. Here $x_{a}=E_{1} / I_{m}$ is the so-called exciting reactance of the machine. Multiplying and dividing the foregoing expression 
by $I_{m}$ we get, torque $=I^{2} x_{a} u \sin \alpha=E_{1} I\left(u I \sin \alpha / I_{m}\right)$. But from the triangle $O A^{\prime} B^{\prime}$ (Fig. 7) we have that $u I / \sin \gamma=$ $I_{m} / \sin \alpha$. Substituting in the preceding equation we find that the torque $=E_{1} I \sin \gamma=A T \cdot I$.

Recapitulation. The motor characteristics (Fig. 7) are essentially determined by the ratio $O A$ to $A B$ of the effective stator and rotor ampere-turns, and by the brush shift angle $\alpha$. The direction of the vector of current $I$ is always along the axis of abscissas, that of the terminal voltage $O K$ approaches $I$ as the speed increases. Select a value of $I$ and plot $O A^{\prime}=I$, remembering that $O A^{\prime}$ does not represent the true direction of $I$ in the time diagram, but only its magnitude. Then the following values are obtained:

Magnetizing current $O B^{\prime}=I_{m}$

Stator voltage $O A=E_{1}$. It is found from Fig. 8 as the ordinate corresponding to abscissa $I_{m}$

Rotor voltage at standstill $A B=E_{2}$

Rotor voltage at $\operatorname{slip} s, A C=s E_{2}$

Total useful voltage at slip $s, O C=E=E_{1}+s E_{2}$ (geometric addition)

Reactive drop $C K^{\prime \prime}=I x$

Ohmic drop (which may be increased to cover core loss) $K^{\prime \prime} K=I r$

Terminal voltage $O K=P=E+I x+I r$

Slip $A^{\prime \prime} C^{\prime \prime}=s$, where $A^{\prime \prime} B^{\prime \prime}=100$ per cent slip

Speed $C^{\prime \prime} B^{\prime \prime}=1-s$, where $A^{\prime \prime} B^{\prime \prime}=100$ per cent synchronous speed.

Input $K K^{\prime} \cdot I$

Output $C C^{\prime} \cdot I$

Efficiency $C C^{\prime} / K K^{\prime}=1-\left(K K^{\prime \prime} / K K^{\prime}\right)$

Power factor $\cos \phi=K K^{\prime} / O K=O L^{\prime}$, where $O Q=O L=$ 100 per cent

'Torque in synchronous watts $A T \cdot I$

\section{E. The Secomor and its Use}

The secomor (Fig. 9) is built out of four flat iron bars; these bars represent the principal vectors in Fig. 7. Each bar is provided with a centimeter scale, and they are mounted on an ordinary drafting board. A sheet of cross-section paper is tacked to the board and serves as a universal scale. The four bars and their functions are as follows: 
(a) The stator bar, $O A$, determines the stator voltage $E_{1}$ and the current $O A^{\prime}=I$ (Fig. 7)

(b) The rotor bar, $D B$, determines the rotor voltages $E_{2}$ and $s E_{2}$.

(c) The speed bar, $D^{\prime \prime} B^{\prime \prime}$, determines the slip $s$ or the speed of the motor.

(d) The setting bar, $C^{\prime \prime} L$ determines the point $C$ for which the useful voltage $E$ plus the impedance drop $C K$ gives the terminal voltage $P=O K$.

The setting bar is pivoted at $O$ next to the cross-section paper, the stator bar is pivoted on top of it, and the other two bars slide on top of the stator bar. On each bar one edge is called the "reading" edge; on the pivoted bars the reading edge passes through the geometric center of the pivot $O$.

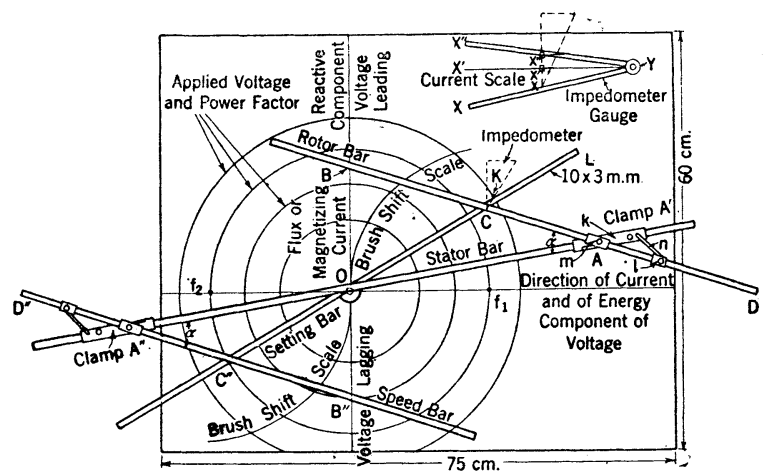

Fig. 9-The Secomor-The Notation Corresponds to Fig. 7

The clamps $A^{\prime}$ and $A^{\prime \prime}$ have to be rather carefully made, to permit of an accurate setting and not to have too much lost motion. The long sleeve $k$ of the clamp slides on the stator bar; the sleeves $l$ and $m$ guide the rotor bar as it slides in them. The sleeve $m$ is pivoted on top of sleeve $k ; l$ is connected to $k$ by means of a pivoted rod $n$. By tightening the set screw on $k$ the rotor bar may be fixed at any desired point of the stator bar. By loosening the set screws on $l$ and $m$ the angle $\alpha$ can be adjusted, and after the screws have been tightened the angle remains constant. Part of each sleeve is cut out to enable one to see the scale and the reading edges of the bars.

It would be inconvenient to use a protractor for setting the rotor bar and the speed bar at a desired brush-shift angle $\alpha$. Therefore, two large circular scales are provided on the base 
of the apparatus, with centers on the axis of abscissas, at $f_{1}$ and $f_{2}$. These scales are marked in degrees and each is labeled in Fig. 9 "brush shift scale". To use the scale $f_{1}$ the stator bar is so turned that its reading edge coincides with the axis of abscissas; the clamp $A^{\prime}$ is shifted until the reading edge of the rotor bar passes through $f_{1}$. Then the rotor bar may be set at any desired angle. After the set screws on $l$ and $m$ have been tightened the angle remains unchanged when the clamp is shifted. The speed bar must be set at the same angle at $f_{2}$, and the two bars must always be parallel to each other when the secomor is in use.

The four concentric circles with the centers at $O$ are used for the applied voltage. Any of them can be selected as a locus of point $K$. With some motor characteristics the bars are not long enough to be used with the largest circle and one has to

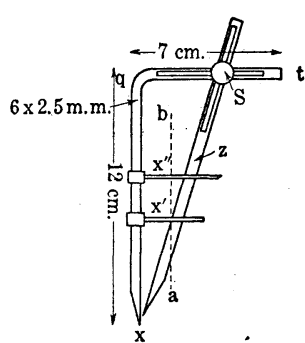

FIg. $10-$ The IMPEDOMETER USED WITH THE SECOMOR FOR TAKING into Account THE IMPEDANCE DROP ous speed, the upper indicator $x^{\prime \prime}$ is set for the value of $I x$ for standstill at the same current. The value of $I x$ for any sub-synchronous speed lies between $x^{\prime}$ and $x^{\prime \prime}$, and for super-synchronous speeds it lies below $x^{\prime}$. These values can be readily estimated by the eye, the whole correction for the impedance drop being small. Point $K$ (Fig. 7) lies somewhere on $a b$ (Fig. 10) but it is not necessary to have this line marked on the impedometer, because the device, when in use is lying on the cross-section paper with $x q$ parallel to one of the rulings (Fig. 9), and the eye easily follows the direction $a b$.

The two diverging straight edges $X$ and $X^{\prime \prime}$ (Fig. 9) shown in the upper right-hand corner of the secomor serve as a guage for a quick setting of the impedometer. The horizontal line $X^{\prime}$ is one of the rulings on the cross-section paper, and serves as a current scale and the locus of the point $x^{\prime}$ of the impedo- 
meter. Bar $X$ is the locus for point $x$, and bar $X^{\prime \prime}$ is the locus for $x^{\prime \prime}$. The set screw $Y$ fixes the bars in any desired position.

The saturation curve (Fig. 8) used with the secomor, is made out of a piece of ordinary solder wire, and can be readily bent and adjusted by hand to any desired shape. The lower end of the wire is held in a swivel clamp at $O$, and the upper end is clamped at $R$. The clamp $R$ can be loosened and moved up and down in the slot $T$. The whole is mounted on a piece of board; a sheet of cross-section paper tacked to it serves as a universal scale.

The method of using the secomor follows directly from Fig. 7 , the setting for different constants and for different loads being accomplished by shifting or turning the four bars. The reader can simply follow the "Recapitulation" above. As to the selection of scales for volts and amperes, the simplest method seems to be always to read everything directly in centimeters and to use constants afterwards. When testing an actual machine one usually reads the meters without regard to their constants, and later recomputes the data.

The use of the impedometer requires no particular skill or precision. Having located point $C$ approximately near the chosen circle of applied voltage, the impedometer and the setting bar are shifted to and fro until the electrical condition for the sum of the voltages is fulfilled. This condition depends upon the speed which is simultaneously read at $C^{\prime \prime}$. Point $K$ must always lie on $a b$ (Fig. 10), but its exact position on $a b$ is determined by the speed of the machine. At standstill it must lie on the intersection of $a b$ with the indicator $x^{\prime \prime}$, at synchronism it lies on the intersection of $a b$ with $x^{\prime}$. At any other speed $K$ divides $x^{\prime} x^{\prime \prime}$ in the same proportion in which $C^{\prime \prime}$ divides $A^{\prime \prime} B^{\prime \prime}$. The length $x^{\prime} x^{\prime \prime}$ being small as compared to $O C$ or $O K$, the judgment of the eye is amply sufficient. An engineer who uses the device regularly will soon find several short cuts which it is not necessary to mention here.

The Effect of a Series Transformer. When the armature of the motor is fed through a series transformer (Fig. 3) the vector diagrams (Figs. 6 and 7 ) become somewhat more involved, and the use of the secomor is accordingly modified.* A series transformer introduces the following new factors:

(a) A magnetizing current and an appreciable saturation in

*For a detailed graphical and analytical treatment of this case see an article by Dreyfus and Hillebrand in the Elektrotechnik und Maschinenbau, Vol. 30 (1912), p. 389. 
the transformer core at speeds remote from the synchronous speed, when the armature voltage is high.

(b) A primary and a secondary leakage impedance, due to the transformer windings.

(c) An iron loss in the transformer core.

(d) A new vectorial difference in time phase between the armature and the field currents, and consequently a new space angle between the primary and secondary $\mathrm{m}$. $\mathrm{m}$. f's, not determined by the brush shift alone.

The equivalent electric diagram which takes the series transformer into account is shown in Fig. 11, for one phase only. First, the actual transformer is replaced by a one-to-one transformer, with the corresponding changes in the constants of the

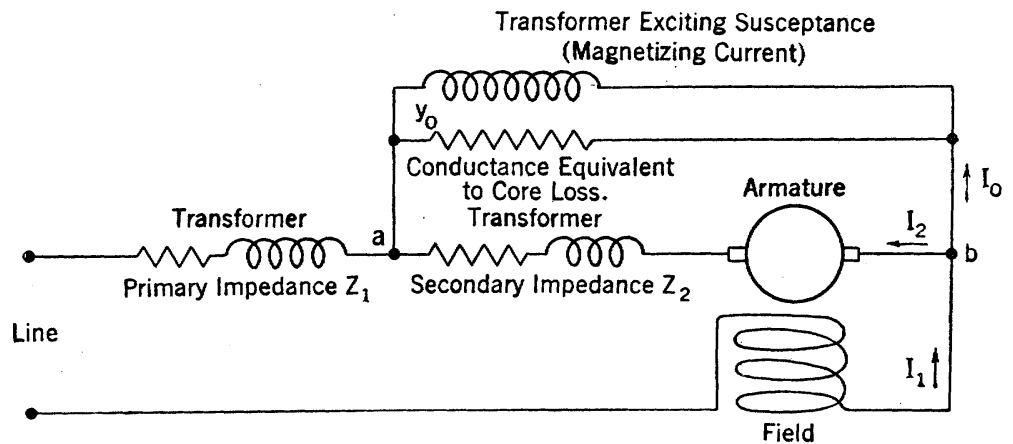

Fig. 11-The Equivalent Diagram of Connections when the Armature is Supplied with Power Through a Transformer, ACCORDING TO FIG. 3

armature circuit. Then this equivalent transformer is removed and an exciting admittance $\gamma_{0}$ is shunted around the armature to provide a path for the magnetizing current of the actual transformer and for its core loss. The resistance of the transformer windings and the effect of the magnetic leakage are taken into account by means of two equivalent impedances, $Z_{1}$ and $Z_{2}$, in the primary and armature circuits respectively. The combination of $\gamma_{0}, Z_{1}$ and $Z_{2}$ is equivalent to the actual transformer in its effect upon the performance of the motor. $\dagger$

The magnetizing current of the transformer is a function of the voltage between the points $a$ and $b$ (Fig. 11), and is supposed

$\dagger$ For the theory of the replacement of a transformer by equivalent mpedances see for example the author's Electric Circuit, Chap. XI. 
to be given in the form of an a-c. saturation curve similar to the saturation curve of the motor itself, shown in Fig. 8. The voltage $E_{a b}$ between $a$ and $b$ is somewhat different from the e. m. f. $s E_{2}$ induced in the armature, the difference being due to the impedance drop between $a$ and $b$. But it is only at speeds far remote from synchronism that $E_{a b}$, and consequently the magnetizing current of the transformer, are of importance. For such speeds the difference between $E_{a b}$ and $s E_{2}$ may be neglected in the first approximation, and the magnetizing current assumed to be a function of the counter e. m. f. $s E_{2}$ of the machine. A

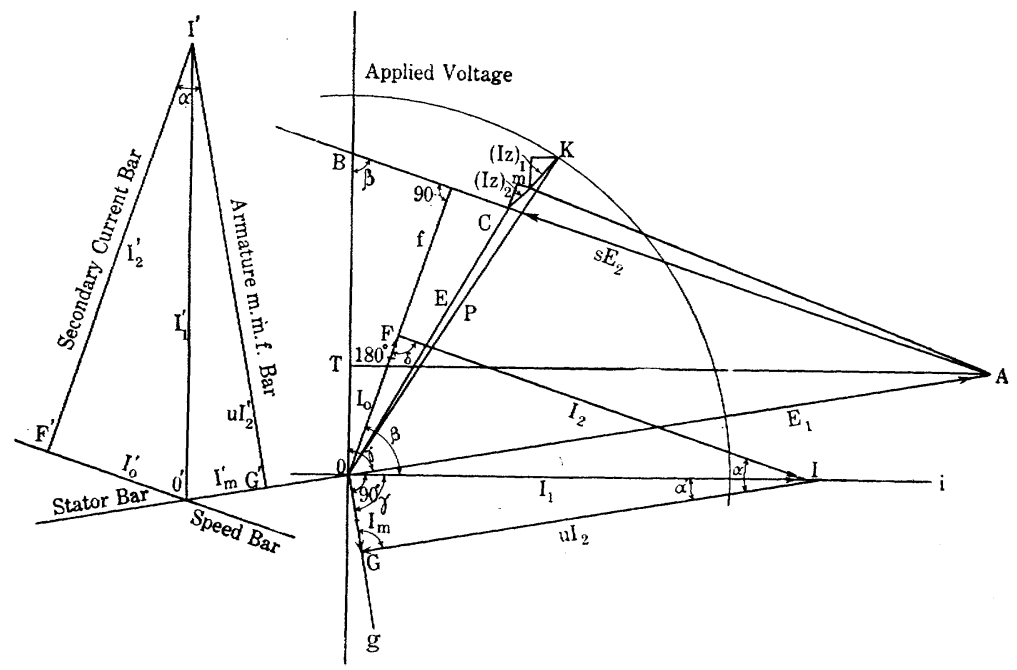

Fig. 12-Vector Diagram of Currents and Voltages, Taking Into Account the Magnetizing CURRent of the Transformer and its IMPEDANCE

correction may be made later, using the approximate values first obtained.

Vector Diagram with Transformer. In Fig. 12, let the triangle $O A B$ represent the same voltages as in Fig. 7, and let $O I$ be the vector of primary current $I_{1}$ which flows through the stator windings. The secondary or armature current $I_{2}$ differs from $I_{1}$ by the amount $I_{0}$ of the magnetizing current in the transformer, as shown in triangle $O F I$. The transformer is supposed to have a one-to-one ratio. According to the approximate assumption made above, $I_{0}$ is a function of the armature e. m.f., a-c., and lags behind it by 90 degrees (neglecting 
the core loss in the transformer). The corresponding corrections will be considered later.

The m. m. f. space triangle which is shown in Fig. 7 in the position $O A^{\prime} B^{\prime}$, is shown in Fig. 12 in the position $O I G$. One should think of its three sides as being multiplied by the number $n_{1}$ of the effective stator turns (Fig. 6). It expresses the fact that the stator ampere-turns $n_{1} I_{1}$ and the armature ampereturns $n_{2} I_{2}=u n_{1} I_{2}$ together give the magnetizing ampereturns $n_{1} I_{m}$ of the machine. The magnetizing current $I_{m}$ is drawn in lagging quadrature with the stator e. m. f. $E_{1}$. When the magnetizing current of the transformer $I_{0}=0$ the m. m.f. triangles in Figs. 7 and 12 become identical, except for their position in the diagram and for the fact that in Fig. 12 the vectors are taken to rotate counter-clockwise in space as well as in time. This is done in order to be able to combine the triangles $O F I$ and $O I G$ into one diagram. To convert the triangle $O A^{\prime} B^{\prime}$ (Fig. 7) into $O I G$ (Fig. 12), first turn $O A^{\prime} B^{\prime}$ in its own plane with $O$ as a center until $A^{\prime}$ lies on $O I$. Then rotate the whole triangle in space about the new axis $O A^{\prime}$ by 180 degrees. Point $B^{\prime}$ will then come into position $G$, and $O B^{\prime}$ will become perpendicular to $O A$.

The angle between the vectors $F I=I_{2}$ and $I G=u I_{2}$ is equal to $180^{\circ}-\alpha$, same as in Fig. 7 , because the vector $F I$ represents $I_{2}$ as a component of the stator current, while $I G$ represents $I_{2}$ as the rotor current, and the brush shift of $\alpha$ degrees is measured from the opposition setting. Angle $\alpha^{\prime}$ in Fig. 12 shows the effect of the magnetizing current in the transformer upon the space phase-angle between the stator and rotor m. m. f's. When $I_{0}=0, \alpha^{\prime}=\alpha$.

Since the directions $O A$ and $A B$ are fixed, the directions $O f$ and $O g$ of the vectors of the two magnetizing currents are also fixed, as well as the direction $O I$. When the load varies, points $G, F$, and $I$ move on their respective loci in such a manner that the angle $F I G$ is always equal to $\alpha$. When this condition is fulfilled the ratio of $I G$ to $I F$ remains equal to $u$. This property of the quadrilateral $O F I G$ is of sufficient importance to warrant a proof.

Lemma. In a variable quadrilateral such as $O F I G$ (Fig. 12) in which the sum of the two opposite angles remains equal to $180^{\circ}$, and the angles $\alpha$ and $180^{\circ}-\alpha$ are constant, the ratio of $I G$ to $I F$ remains constant when the three vertices $I, F, G$, move in an arbitrary manner on the three loci $O i, O f$, and $O g$. 
Proof: If the sum of the angles at $O$ and $I$ is equal to $\pi$, then the sum of the angles at $G$ and $F$ must also be equal to $\pi$, and we have:

from which

$$
\frac{I_{1}}{\sin \delta}=\frac{u I_{2}}{\sin \gamma}=\frac{I_{2}}{\sin \beta}
$$

$$
u=\frac{\sin \gamma}{\sin \beta}
$$

With the motion of the three vertices as specified above, the angles $\beta$ and $\gamma$ remain constant, so that $u$ also remains constant, and this proves the theorem.

This value of $u$ is the same as that used in the construction of the voltage triangle $O A B$, because from this triangle we have

$$
\frac{\sin \gamma}{\sin \beta}=\frac{A B}{O A}=\frac{u E_{1}}{E_{1}}=u \text {. }
$$

Transformer Attachment. In the secomor, it would be rather inconvenient to use the quadrilateral of currents $O F I G$ in its true position, because the rods $O F$ and $O G$ would have to move perpendicularly to $A B$ and $O A$ respectively. For this reason he quadrilateral is turned by 90 degrees into the position $O^{\prime} G^{\prime} I^{\prime} F^{\prime}$, in order that the two magnetizing currents, $I^{\prime}{ }_{m}$ and $I^{\prime}{ }_{0}$ may be represented by certain lengths $O^{\prime} G^{\prime}$ and $O^{\prime} F^{\prime}$ on the stator bar and the speed bar. The cross-section paper provides the direction $O^{\prime} I^{\prime}$, so that only two new members or rods are needed, viz., to represent $I^{\prime}{ }_{2}$ and $u I^{\prime}{ }_{2}$.

The actual construction of the transformer attachment is shown in Fig. 13; it is fastened to the secomor board (Fig. 9) in the upper left-hand corner. Two strips of brass are used to represent the sides $I^{\prime} F^{\prime}$ and $I^{\prime} G^{\prime}$ of the quadrilateral. They will be referred to as the secondary current bar and the armature m. $m$. $f$. bar respectively. These bars are pivoted together on a setscrew $I^{\prime}$ and the directions of their inner edges pass through the center of $I^{\prime}$. The bars may be set at any desired angle by means of the graduated scale $G$ and setscrew $S^{\prime}$. The position of $I^{\prime}$ on the board may be varied by moving the iron $\operatorname{rod} R$ in the clamp $C$, and by moving the clamp itself along the edge of the board. $S$ is a setscrew which holds $R$ on $C$.

To illustrate the use of the transformer attachment, let us assume that the performance characteristics are desired at a given total current $I_{1}$ with a given terminal voltage. Let the 
impedance drop (Fig. 12) be at first neglected so that the counter e. $\mathrm{m}$. f. $O C=E$ is equal to the applied voltage $O K=P$. In the transformer attachment to the left, set the pivot $I^{\prime}$ at the proper point corresponding to the given length $O^{\prime} I^{\prime}=I_{1}$, and spread the rods $I^{\prime} F^{\prime}$ and $I^{\prime} G^{\prime}$ to the proper angle $\alpha$. Select a reasonable arbitrary position of point $K$ on the quadrant of applied voltage. This will give some tentative values of the stator voltage $E_{1}=O A$ and of the armature voltage $C A=K A$ $=s E_{2}$. From the saturation curve of the motor (Fig. 8) the

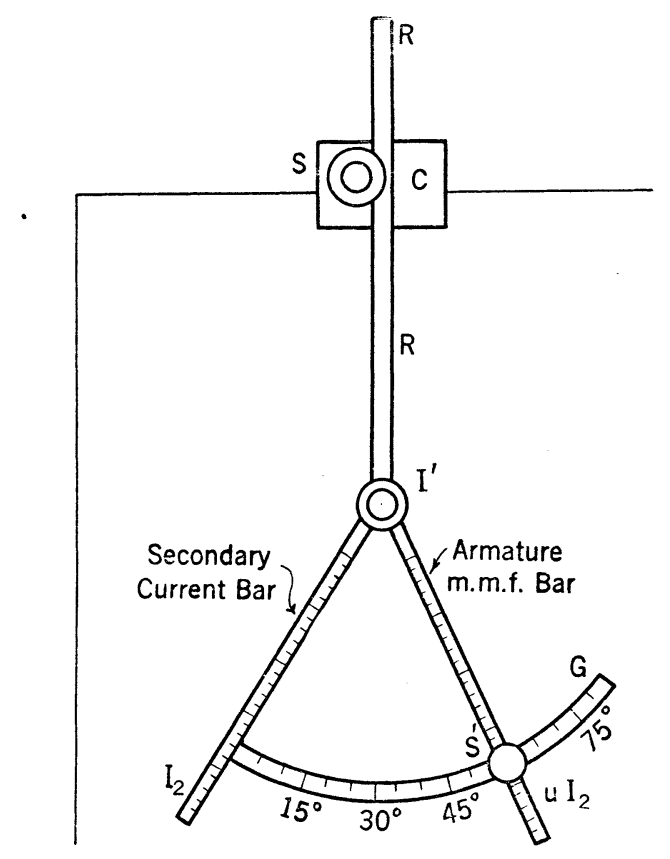

Fig. 13-The Transformer Attachment to the Secomor

magnetizing current $I_{m}$ is found, and its value is marked on the stator bar as $O^{\prime} G^{\prime}$ by bringing the armature m. m. f. bar $I^{\prime} G^{\prime}$ to point $G^{\prime}$. Similarly, the transformer magnetizing current $I_{0}$ corresponding to $s E_{2}$ is found from its saturation curve; it must check with the value $O F^{\prime}$ indicated by the secondary current bar at $F^{\prime}$. If it does not check, the point $K$ was guessed at wrong and a new trial is necessary. After a few trials, the correct position of $C$ or $K$ is found, and this enables one to find the slip, the power factor, the torque and the output of the machine, in the same manner as without the transformer. 
The Torque. The expression for the torque is not modified by the magnetizing current of the transformer, if we neglect the core loss. Since $I_{0}$ is in quadrature with $E_{2}$, the components of $I_{1}$ and of $I_{2}$ in phase with $E_{2}$ are equal to each other. Thus, either with or without the transformer the true input into the field at standstill is equal to the armature output. Both are a measure for the torque in synchronous watts, and the expression $A T \cdot I_{1}$, deduced above, holds true in this case, with the machine running or standing still. In the language of the Vector Analysis, using the dot for the inner or scalar product, we have:

$$
\begin{gathered}
I_{1}=I_{0}+I_{2} \\
\left(E_{1}+E_{2}\right) \cdot I_{1}=O
\end{gathered}
$$

Multiplying the first equation by $E_{2} \cdot$ we get

$$
E_{2} \cdot I_{1}=E_{2} \cdot I_{0}+E_{2} \cdot I_{2}
$$

But $E_{2} \cdot I_{0}=0$, and combining the second and the third equations we get

$$
E_{1} \cdot I_{1}+E_{2} \cdot I_{2}=0 .
$$

This means that the two ideal wattmeter readings are equal to each other, and either one is a measure for the torque in synchronous watts.

Correction for Impedance. The impedance drop in the motor and in the transformer (Fig. 11) may be divided into two parts:

(a) That due to current $I_{1}$ in the stator winding and in the transformer primary;

(b) That due to the current $I_{2}$ in the motor armature and in the transformer secondary.

Consequently, two impedance triangles are necessary, as is shown in Fig. 12 between points $C$ and $K$. The long side of one is parallel to $I^{\prime}{ }_{1}$, that of the other to $I^{\prime}{ }_{2}$. In many cases it will be permissible to neglect the difference between $I_{1}$ and $I_{2}$ and to use a single impedometer (Fig. 10) for an intermediate value of the current, but if a greater accuracy is required, a double impedometer could be constructed, or a single impedometer applied twice.

We shall assume now that the point $K$ has been tentatively assumed on the quadrant of applied voltage, and then the impedometer applied to find the point $C$. This gives the position of point $A$ and consequently the speed and the magnetizing current $I_{m}$ of the motor. The magnetizing current $I_{0}$ of the transformer has been assumed above to be a function of the induced voltage 
$C A=s E_{2}$ in the armature. In reality it is a function of the voltage between points $a$ and $b$ (Fig. 11). This voltage is equal to $A_{m}$ (Fig. 12), being a sum of the e. m. f. $A C$ and the primary impedance drop $C \mathrm{~m}$. Thus, a more accurate value of $I_{0}$ may be taken from the saturation curve. Strictly speaking the direction of the new $I^{\prime}{ }_{0}$ in the transformer attachment should be parallel to $A m$ and not along the speed bar which is parallel to $A C$. This small correction is best made by the judgment of the eye, without disturbing the bars.

If desired, a correction may also be made for the core loss in the transformer, as is indicated in Fig. 11 by an "exciting conductance". This means the addition to $I_{0}$ of a component in phase with $A C$, or more accurately, in phase with $A \mathrm{~m}$. Similarly, the current $I_{m}$ may be corrected to account for the stator core loss.

It would complicate the mathematical theory of the motor very much if one attempted to take all these refinements into account accurately. A kinematic attachment for this purpose would also be unnecessarily involved for most practical purposes. The device as described above gives a nearly accurate solution with a simple setting; small corrections are best applied by the judgment of the eye from this setting. The transformer attachment has a valuable property expressed by the lemma proven above, as long as the sum of its opposite angles is kept equal to $180^{\circ}$. The exact values for the transformer magnetizing current and for the core losses would destroy this valuable property without an adequate advantage in the accuracy of the results achieved.

\section{Conclusion}

In presenting the secomor to the electrical profession the author wishes to point out the possibility of predicting the performance of the polyphase series commutator motor by means of a kinematic device. He also hopes to arouse interest in the use of similar kinematic devices for the prediction and analysis of performance of other types of electrical machinery. Besides the secomor, he has designed the "shucomor" or a kinematic device which imitates the performance of a shunt-wound polyphase commutator motor, and as a by-product has obtained a device which gives the performance of the ordinary induction motor. Alternator characteristics can probably be imitated in a similar manner as well as those of single-phase commutating machines.

The usefulness of such devices is not limited to a-c. machinery, 
but embraces special cases of d-c. machinery as well. Sometime ago the author became interested in the operation of the Entz electromagnetic clutch and transmission used in some gasoline motor cars. He has built a kinematic device which imitates the performance of this ingenious clutch at different engine speeds and with any setting of the regulating resistances. The saturation curves of the two d-c. machines are also incorporated in the device. The device was demonstrated at the A. I. E. E. meeting in Syracuse, N. Y., in May 1917, and has been presented by the author to the Electrical Engineering Department of Syracuse University.

A kinematic device can no more replace human intelligence than a formula or a vector diagram can. But a mechanical device helps and guides an engineer's judgment, and makes it possible to achieve results with less time and trouble. One working on the design of the same type of electrical devices year after year finally attains some proficiency and needs but little outside help. But industrial efficiency demands that younger men do at least routine designing without much previous experience. A mechanical device makes it safer to entrust them with the determination of dimensions, because it enables them readily to check the performance. The effort of more gifted and mature engineers may thus be devoted to new developments and to large important problems, and less of their time need be occupied by the supervision over younger men. A wide use of mechanical devices that imitate the performance of electrical machinery is thus a step in the desired direction.

\section{BIBLIOGRAPHY}

The subject is covered quite thoroughly up to 1910 in E. Arnold's "Die Wechselstromtechnik," Vol. 5, part 2. The theory of both the series and the shunt-wound polyphase commutator motor has been principally studied in Germany, Austria, Switzerland and Sweden, and a few electric manufacturing companies in those countries have put such motors on the market. In France M. Latour has built some 10-pole motors as large as 500 h.p., with the armature connected 12-phase, and with only one stud of brushes per pole, using a two-circuit winding. A brief note on these motors will be found in La Lumiére Electrique, Vol. 29, p. 289.

A person interested in some phase of the subject should refer to the last volumes of the principal German and French electrical periodicals and search through the back volumes "counter-clockwise," until he has found the particular bit of information sought. For such a search the entry words in the indexes are probably much more valuable than a specific reference to a few articles. An entry word will enable the future 
investigator to locate in time articles yet unwritten. The magazines below are arranged in the order of their importance for the particular topic under discussion. The first two periodicals contain by far the largest amount and the best information. Of the American periodicals, The General Electric Review so far has been the only one in which original articles on the polyphase commutator motor have been published. Science Abstracts (London), part B, Electrical Engineering, is a most useful help in literature search on most any electrical subject.

Name of Periodical

Elektrotechnische '?eitschrift

Elektrotechnik und Maschinenbau

Archiv für Elektrotechnik

General Electric Review

La Lumière Electrique*
Entry Word in the Index

Elektromotoren

Kollektor, Kommutator.

Mehrphasen

Name index, see Glossary below

Motors

Machines, Moteurs

Glossary of German and French Terms for Facilitating Literature Search

\begin{tabular}{|c|c|c|}
\hline $\begin{array}{l}\text { English } \\
\text { machine }\end{array}$ & $\begin{array}{c}\text { French } \\
\text { machine }\end{array}$ & $\begin{array}{l}\text { German } \\
\text { Maschine }\end{array}$ \\
\hline motor & moteur & Motor or Elektromotor \\
\hline single-phase & monophasé & Einphasen or Wechselstrom \\
\hline two-phase & diphasé & Zweiphasen \\
\hline three-phase & triphasé & Drehstrom \\
\hline commutator & collecteur & Kommutator or Kollektor \\
\hline series-wound & série & Reihenschluss or Serien \\
\hline shunt-wound & shunt & Nebenschluss \\
\hline
\end{tabular}

